\title{
Effect of Integrated Nutrient Management on Growth, Yield and Quality of Carrot (Daucus carota L.) cv. New Kuroda
}

\author{
*Varun Shekhar; **Dr. Devi Singh; ***Mr. Deepanshu \\ *Research Scholar, Department of Horticulture (Vegetable Science), SHUATS, Prayagraj \\ **Assistant Professor, Department of Horticulture, SHUATS, Prayagraj \\ ***Assistant Professor, Department of Horticulture, SHUATS, Prayagraj \\ DOI: 10.47856/ijaast.2021.v08i10.003
}

\begin{abstract}
The experiment entitled "Effect of Integrated Nutrient Management on Growth, Yield and Quality of Carrot (Daucus carota L.) cv. New Kuroda" was conducted during Rabi season of the year 2020-2021 on Department of Horticulture, Sam Higginbottom University of Agriculture, Technology, Prayagraj. The experiment was laid out in Randomized Block Design comprising of 13 treatments each replicated three times. Treatments were randomly arranged in each replication, divided into thirty nine plots. The experiment was carried out with the thirteen integrated nutrient management treatments. Among the treatments, $\mathrm{T}_{11}$ FYM $10 \mathrm{t}$ ha-1+Vermicompost 2.5t ha-1 + Biofertilizer (2 kg ha-1) 75\% NPK registered significantly higher plant height (82.95), number of leaves per plant (14.66), root length (10.82), root diameter (3.42), fresh weight of root (67.01), dry weight of root (4.13), root yield $\operatorname{plot}^{-1}(17.80 \mathrm{~kg})$, root yield $\left(455.00 \mathrm{q} \mathrm{ha}{ }^{-1}\right)$ and Total soluble solid $\left(9.45^{0}\right.$ Brix). Whereas the maximum benefit cost ratio (1: 5.24 \& 5.29) was found in $\mathrm{T}_{10}$ and $\mathrm{T}_{12}$.
\end{abstract}

Keywords:- Integrated nutrient management, Carrot, Growth, Yield and quality. 


\section{$\vec{x}$}

Varun Shekhar et al, International Journal of Advances in Agricultural Science \& Technology,

Vol.8 Issue.10, October-2021, pg. 17-28

ISSN: 2348-1358

Impact Factor: 6.057

NAAS Rating: 3.77

\section{INTRODUCTION}

Carrot (Daucus carota $\mathrm{L}$.) is an important root vegetable, belongs to the family umbelliferae with diploid chromosome number $2 \mathrm{n}=18$. It is cultivated all over the world, during spring-summer in temperate countries and winter in tropical and subtropical countries. Carrot is an excellent source of carotene a precursor of vitamin A and fibre in the diet (Handelman, 2001).

Daucus carota is a biennial plant in the Umbellifer family Apiaceae. Fast- growing cultivars mature within three months (90 days) of sowing the seed, while late maturing cultivars are harvested four months later (120 days).After germination, carrot seedlings show a distinct demarcation between taproot and leaf.

Carrot plant is an erect, biennial plant. Leaves have long petiole compound and pinnate (Kochhar, 2011).

Carrot cultivars are majorly of two groups, eastern carrots and western carrots (Grubben, 2016). Eastern carrots that survive to the present day are commonly purple or yellow, and often have branched roots. The purple colour common in these carrots comes from anthocyanin pigments (Tiwari et al., 2012).

\section{MATERIALS AND METHODS}

\section{1- Experimental site:}

The experiment entitled "Effect of Integrated Nutrient Management on Growth, Yield and Quality of Carrot (Daucus carota L.) cv. New Kuroda" was conducted during Rabi season of the year 2020-2021 on Department of Horticulture, Sam Higginbottom University of Agriculture, Technology, Prayagraj..

\section{2- Climate and weather condition:}

Prayagraj has subtropical climate, which prevails in the South East part of U.P., with the both extremes of temperature i.e. the winters and the summers. In fairly cold winters (during Oct-Feb), the temperature falls to $3-4^{0} \mathrm{C}$, sometimes below $-1^{0} \mathrm{C}$. During summer (March-June), the temperature rises 
Varun Shekhar et al, International Journal of Advances in Agricultural Science \& Technology,

Vol.8 Issue.10, October-2021, pg. 17-28

ISSN: $2348-1358$ Impact Factor: 6.057

NAAS Rating: 3.77

upto $45^{\circ} \mathrm{C}$, sometimes $47-48^{\circ} \mathrm{C}$ with low relative humidity (20\%) and dust laden winds. During monsoon (June-Sept) $85 \%$ of average rainfall of $1100 \mathrm{~mm}$ with fall in temperature $40-45^{\circ} \mathrm{C}$ on rainy days.

\section{RESULTS AND DISCUSSION}

The present investigation entitled "Effect of Integrated Nutrient Management on Growth, Yield, and Quality of Carrot (Daucus carota L.) cv. New Kuroda" was undertaken at the Department of Horticulture, Naini Agriculture Institute, SHUATS, Praygraj during the rabi season 2020-2021, to find out the most suitable treatment for plants growth, root yield and quality parameters of carrot have been represented with tables and bars-diagrams wherever required.

\section{Effect of Integrated Nutrient Management on Plant height (cm) of carrot (daucus carotal.) cv. New Kuroda}

In the present study, plant height as influenced by integrated nutrient management was significant results at the successive stages of growth till harvest. The statistically analysed data are presented in table 4.1 and graphically shown in fig.4.1.

Effect of integrated nutrient management had shown significant difference on plant height at 30, 60 days and 90 days after sowing. 
Varun Shekhar et al, International Journal of Advances in Agricultural Science \& Technology,

Vol.8 Issue.10, October-2021, pg. 17-28

ISSN: 2348-1358 Impact Factor: 6.057 NAAS Rating: 3.77

Table 4.1 Effect of integrated nutrient management on plant height (cm) of carrot (daucus carotal.) cv. New Kuroda

\begin{tabular}{|c|c|c|c|c|}
\hline \multirow{2}{*}{ Treatments No. } & \multirow{2}{*}{ Treatments Symbols } & \multicolumn{3}{|c|}{ Plant height (cm) } \\
\hline & & 30 DAS & 60 DAS & 90 DAS \\
\hline $\mathrm{T}_{0}$ & Control & 12.07 & 21.47 & 55.14 \\
\hline $\mathrm{T}_{1}$ & FYM 20t ha-1 & 14.90 & 26.18 & 60.94 \\
\hline $\mathrm{T}_{2}$ & Vermicompost $5 \mathrm{t}$ ha- 1 & 15.67 & 27.32 & 66.23 \\
\hline $\mathrm{T}_{3}$ & FYM 10 t ha-1 + Vermicompost 2.5 t ha-1 + NPK $25 \%$ & 16.96 & 28.06 & 62.02 \\
\hline $\mathrm{T}_{4}$ & FYM 10 t ha-1+50\% NPK+ Biofertilizer (2kg ha-1) & 15.48 & 30.36 & 64.10 \\
\hline $\mathrm{T}_{5}$ & Vermicompost $2.5 \mathrm{t}$ ha-1+50\%NPK Biofertilizer (2 kg ha-1) & 17.23 & 32.08 & 70.24 \\
\hline $\mathrm{T}_{6}$ & FYM 10 t ha-1+ 75\% NPK + Biofertilizer (2kg ha-1) & 17.80 & 33.51 & 71.61 \\
\hline $\mathrm{T}_{7}$ & Vermicompost 2.5 t ha-1 + Biofertilizer (2 kg ha-1) & 18.90 & 33.32 & 71.81 \\
\hline $\mathrm{T}_{8}$ & FYM 10 t ha-1+Vermicompost 2.5t ha-1 + Biofertilizer (2 kg ha-1) & 19.31 & 31.30 & 79.58 \\
\hline $\mathrm{T}_{9}$ & Vermicompost $2.5 \mathrm{t}$ ha- $1+$ Biofertilizer $(2 \mathrm{~kg}$ ha- 1$)+75 \% \mathrm{NPK}$ & 20.17 & 30.99 & 69.62 \\
\hline $\mathrm{T}_{10}$ & Vermicompost 2.5 t ha-1+ NPK50\% + Biofertilizer (2 kg ha-1) & 19.43 & 29.75 & 79.96 \\
\hline $\mathrm{T}_{11}$ & $\begin{array}{l}\text { FYM } 10 \text { t ha-1+Vermicompost } 2.5 \mathrm{t} \text { ha-1 + Biofertilizer ( } 2 \mathrm{~kg} \text { ha-1) } \\
75 \% \text { NPK }\end{array}$ & 22.88 & 36.98 & 82.95 \\
\hline \multirow[t]{5}{*}{$\mathrm{T}_{12}$} & $100 \%$ NPK (60:80:75 kg ha-1) & 17.37 & 26.99 & 77.11 \\
\hline & F-Test & $\mathbf{S}$ & $\mathbf{S}$ & $\mathbf{S}$ \\
\hline & C.D. at $0.5 \%$ & 2.552 & 3.683 & 2.834 \\
\hline & S.Ed. $( \pm)$ & 1.236 & 1.784 & 1.373 \\
\hline & $\mathbf{C V}$ & 8.662 & 7.313 & 2.399 \\
\hline
\end{tabular}


Varun Shekhar et al, International Journal of Advances in Agricultural Science \& Technology,

Vol.8 Issue.10, October-2021, pg. 17-28

ISSN: $2348-1358$ Impact Factor: 6.057

NAAS Rating: 3.77

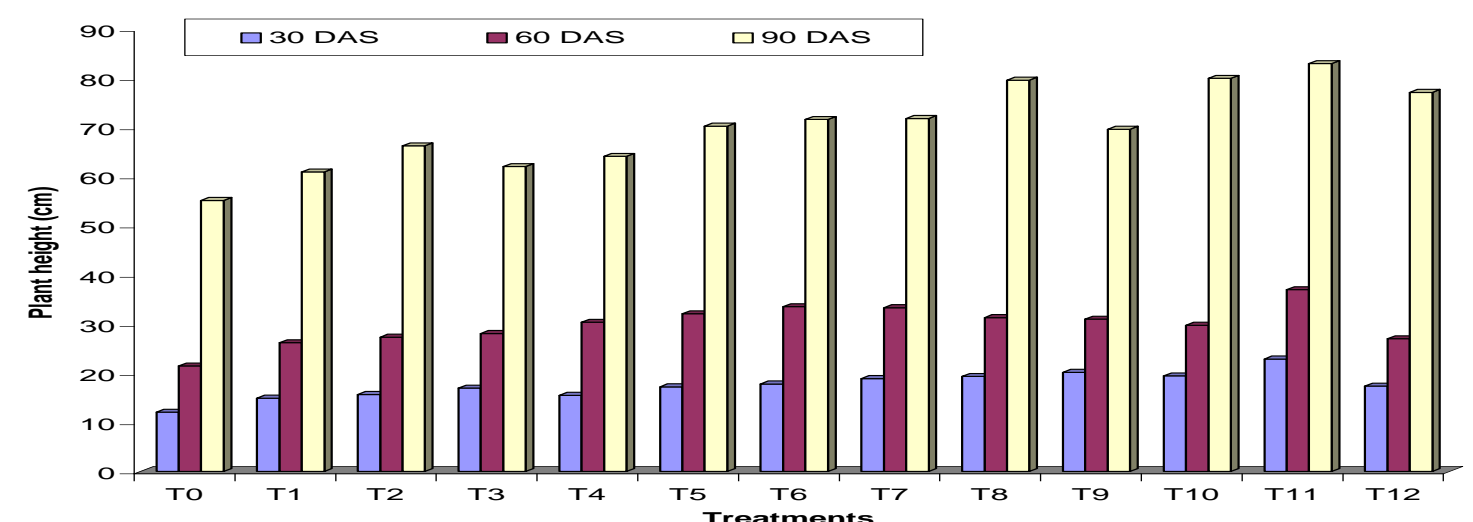

Fig. 4.1 Effect of integrated nutrient management on plant height (cm) of carrot (daucus carotal.) cv. New Kuroda

2. Effect of integrated nutrient management on root length (cm) of carrot (daucus carota L.) cv. New Kuroda

Effect of integrated nutrient management had shown significant difference on the root length of carrot at harvest. The statistically analysed data have been presented in the table 4.2 and graphically shown in the fig. 4.2 .

The maximum root length (23.48) was recorded with treatment $\mathrm{T}_{11}$ FYM $10 \mathrm{t}$ ha-1+Vermicompost 2.5t ha-1 + Biofertilizer (2 kg ha-1) 75\% NPK.Whereas the minimum root length (10.82) was obtained with the treatment $\mathrm{T}_{1}$ (Control). 
Varun Shekhar et al, International Journal of Advances in Agricultural Science \& Technology,

Vol.8 Issue.10, October-2021, pg. 17-28

ISSN: 2348-1358 Impact Factor: 6.057 NAAS Rating: 3.77

Table 4.2 Effect of integrated nutrient management on root length (cm) of carrot (daucus carota L.) cv. New Kuroda

\begin{tabular}{|c|c|c|}
\hline Treatments No. & Treatments Symbols & Root length $(\mathrm{cm})$ \\
\hline $\mathrm{T}_{0}$ & Control & 10.82 \\
\hline $\mathrm{T}_{1}$ & FYM 20t ha-1 & 12.57 \\
\hline $\mathrm{T}_{2}$ & Vermicompost $5 \mathrm{t}$ ha-1 & 15.38 \\
\hline $\mathrm{T}_{3}$ & FYM 10 t ha-1 + Vermicompost $2.5 \mathrm{t}$ ha- $1+$ NPK $25 \%$ & 14.93 \\
\hline $\mathrm{T}_{4}$ & FYM 10 t ha-1+50\% NPK+ Biofertilizer (2kg ha-1) & 16.82 \\
\hline $\mathrm{T}_{5}$ & Vermicompost $2.5 \mathrm{t}$ ha-1+50\%NPK Biofertilizer (2 kg ha-1) & 18.03 \\
\hline $\mathrm{T}_{6}$ & FYM 10 t ha-1+ 75\% NPK + Biofertilizer (2kg ha-1) & 18.95 \\
\hline $\mathrm{T}_{7}$ & Vermicompost $2.5 \mathrm{t}$ ha-1 + Biofertilizer (2 kg ha-1) & 19.97 \\
\hline $\mathrm{T}_{8}$ & FYM 10 t ha-1+Vermicompost 2.5t ha-1 + Biofertilizer (2 kg ha-1) & 20.74 \\
\hline $\mathrm{T}_{9}$ & Vermicompost $2.5 \mathrm{t}$ ha-1 + Biofertilizer $(2 \mathrm{~kg}$ ha- 1$)+75 \% \mathrm{NPK}$ & 17.63 \\
\hline $\mathrm{T}_{10}$ & Vermicompost $2.5 \mathrm{t}$ ha-1+ NPK50\% + Biofertilizer (2 kg ha-1) & 16.33 \\
\hline $\mathrm{T}_{11}$ & FYM 10 t ha-1+Vermicompost $2.5 \mathrm{t}$ ha-1 + Biofertilizer (2 kg ha-1) $75 \%$ NPK & 23.48 \\
\hline \multirow[t]{5}{*}{$\mathrm{T}_{12}$} & $100 \%$ NPK (60:80:75 kg ha-1) & 20.10 \\
\hline & F-Test & $\mathbf{S}$ \\
\hline & C.D. at $0.5 \%$ & 1.073 \\
\hline & S.Ed. (立) & 0.520 \\
\hline & CV & 3.666 \\
\hline
\end{tabular}


Varun Shekhar et al, International Journal of Advances in Agricultural Science \& Technology,

Vol.8 Issue.10, October-2021, pg. 17-28

ISSN: $2348-1358$

Impact Factor: 6.057

NAAS Rating: 3.77

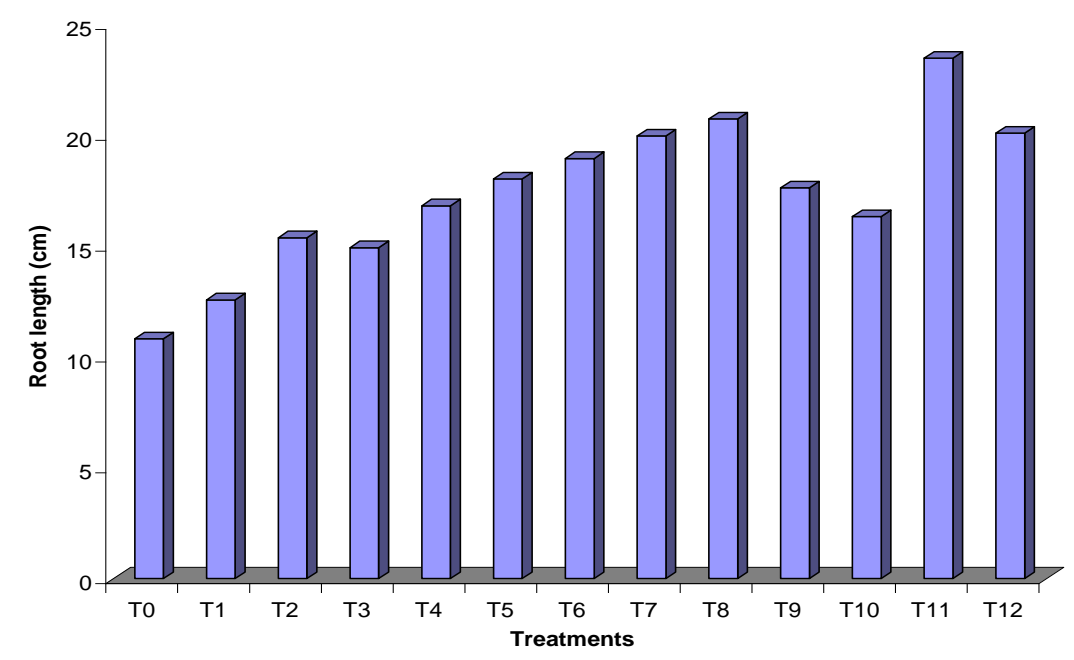

Fig. 4.2 Effect of integrated nutrient management on root length (cm) of carrot (daucus carota L.) cv. New Kuroda

\section{Effect of integrated nutrient management on Root yield plot $^{-1}(\mathrm{~kg})$ of carrot (daucus carota L.) cv. New Kuroda}

Effect of integrated nutrient management had shown significant difference on the root yield $\operatorname{plot}^{-1}(\mathrm{~kg})$ of carrot at harvest. The statistically analysed data have been presented in the table 4.3 and graphically shown in the fig. 4.3 .

The maximum root yield $\operatorname{plot}^{-1}(17.80 \mathrm{~kg})$ was recorded with treatment $T_{11}$ Whereas the minimum root yield plot ${ }^{-1}(10.72 \mathrm{~kg})$ was obtained with the treatment $\mathrm{T}_{1}$ (Control). 
Varun Shekhar et al, International Journal of Advances in Agricultural Science \& Technology,

Vol.8 Issue.10, October-2021, pg. 17-28

ISSN: 2348-1358 Impact Factor: 6.057 NAAS Rating: 3.77

Table 4.3 Effect of integrated nutrient management on Root yield plot $^{-1}(\mathrm{~kg})$ of carrot (daucus carota $\mathrm{L}$.) cv. New Kuroda

\begin{tabular}{|c|c|c|}
\hline Treatments No. & Treatments Symbols & Root yield plot ${ }^{-1}(\mathrm{~kg})$ \\
\hline $\mathrm{T}_{0}$ & Control & 10.72 \\
\hline $\mathrm{T}_{1}$ & FYM 20t ha-1 & 11.70 \\
\hline $\mathrm{T}_{2}$ & Vermicompost $5 \mathrm{t}$ ha-1 & 13.04 \\
\hline $\mathrm{T}_{3}$ & FYM 10 t ha-1 + Vermicompost 2.5 t ha-1 + NPK $25 \%$ & 13.34 \\
\hline $\mathrm{T}_{4}$ & FYM 10 t ha-1+50\% NPK+ Biofertilizer (2kg ha-1) & 14.40 \\
\hline $\mathrm{T}_{5}$ & Vermicompost $2.5 \mathrm{t}$ ha-1+50\%NPK Biofertilizer (2 kg ha-1) & 13.82 \\
\hline $\mathrm{T}_{6}$ & FYM 10 t ha-1+ 75\% NPK + Biofertilizer (2kg ha-1) & 13.49 \\
\hline $\mathrm{T}_{7}$ & Vermicompost $2.5 \mathrm{t}$ ha-1 + Biofertilizer (2 kg ha-1) & 15.02 \\
\hline $\mathrm{T}_{8}$ & FYM 10 tha-1+Vermicompost 2.5t ha-1 + Biofertilizer (2 kg ha-1) & 16.83 \\
\hline $\mathrm{T}_{9}$ & Vermicompost 2.5 t ha-1 + Biofertilizer (2 kg ha- 1$)+75 \% \mathrm{NPK}$ & 16.29 \\
\hline $\mathrm{T}_{10}$ & Vermicompost $2.5 \mathrm{t}$ ha-1+ NPK50\% + Biofertilizer (2 kg ha-1) & 16.49 \\
\hline $\mathrm{T}_{11}$ & FYM 10 t ha-1+Vermicompost 2.5t ha-1 + Biofertilizer (2 kg ha-1) 75\% NPK & 17.80 \\
\hline \multirow[t]{5}{*}{$\mathrm{T}_{12}$} & 100\% NPK (60:80:75 kg ha-1) & 15.12 \\
\hline & F-Test & $\mathrm{S}$ \\
\hline & C.D. at $0.5 \%$ & 1.207 \\
\hline & S.Ed. $( \pm)$ & 0.585 \\
\hline & CV & 4.799 \\
\hline
\end{tabular}


Varun Shekhar et al, International Journal of Advances in Agricultural Science \& Technology,

Vol.8 Issue.10, October-2021, pg. 17-28

ISSN: 2348-1358 Impact Factor: 6.057

NAAS Rating: 3.77

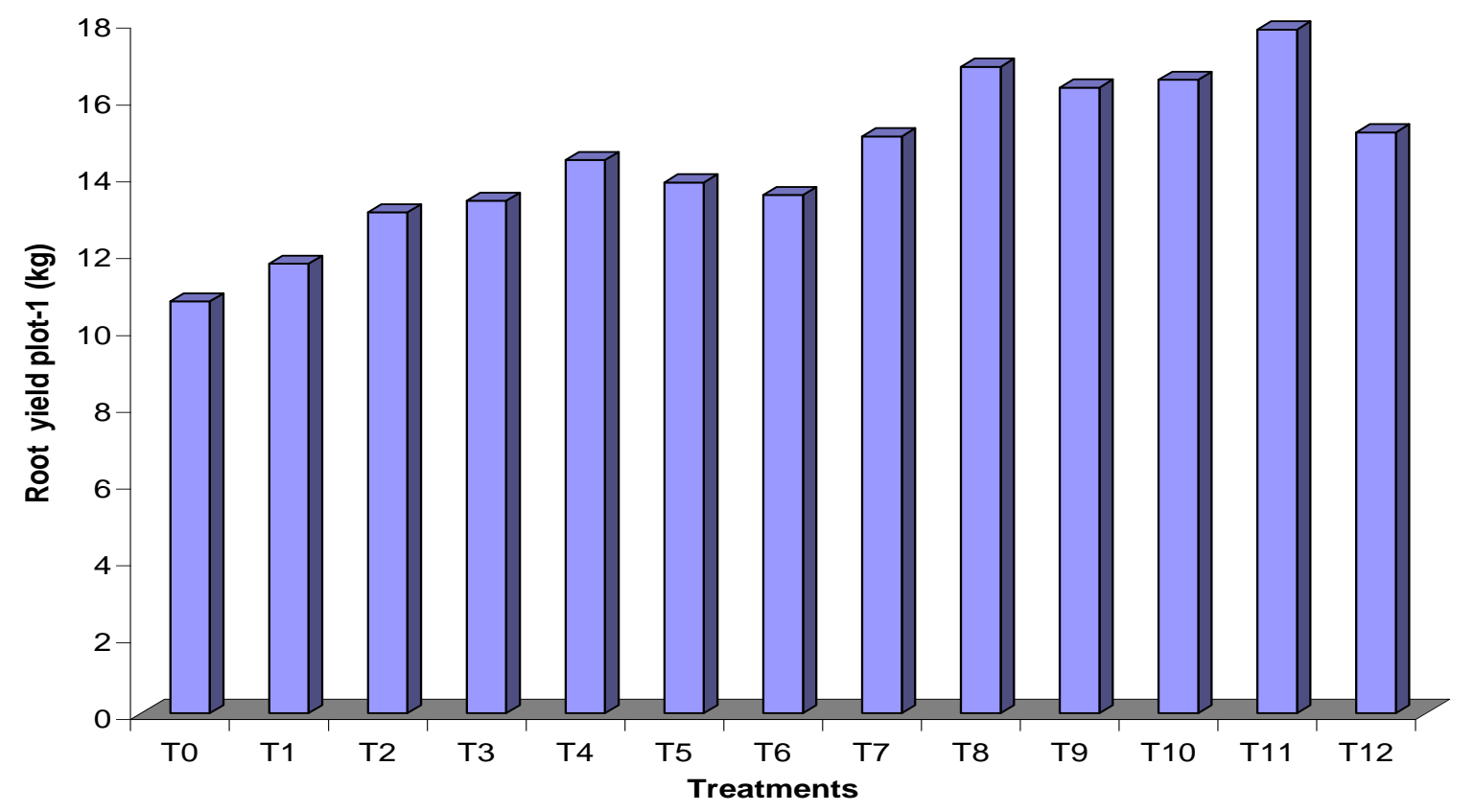

Fig. 4.3 Effect of integrated nutrient management on Root yield $\operatorname{plot}^{-1}(\mathrm{~kg})$ of carrot (daucus carota L.) cv. New Kuroda

\section{Effect of integrated nutrient management on TSS of carrot (daucus carota L.) cv. New Kuroda}

Effect of integrated nutrient management had shown significant difference on the TSS of carrot at harvest. The statistically analysed data have been presented in the table 4.4 and graphically shown in the fig. 4.4 
Varun Shekhar et al, International Journal of Advances in Agricultural Science \& Technology,

Vol.8 Issue.10, October-2021, pg. 17-28

ISSN: 2348-1358 Impact Factor: 6.057 NAAS Rating: 3.77

The maximum total soluble solid $\left(9.45{ }^{0} \mathrm{Brix}\right)$ was recorded with treatment $\mathrm{T}_{11}$, Whereas the minimum total soluble solid ( $\left.{ }^{0} \mathrm{Brix}\right)$ was obtained with the treatment $\mathrm{T}_{1}$ (Control)

Table 4.4Effect of integrated nutrient management on TSS of carrot (daucus carota L.) cv. New Kuroda

\begin{tabular}{|c|c|c|}
\hline Treatments No. & Treatments Symbols & TSS \\
\hline $\mathrm{T}_{0}$ & Control & 6.64 \\
\hline $\mathrm{T}_{1}$ & FYM 20t ha-1 & 8.67 \\
\hline $\mathrm{T}_{2}$ & Vermicompost 5 t ha- 1 & 9.15 \\
\hline $\mathrm{T}_{3}$ & FYM 10 t ha- $1+$ Vermicompost 2.5 t ha-1 + NPK $25 \%$ & 8.86 \\
\hline $\mathrm{T}_{4}$ & FYM 10 tha-1+50\% NPK+ Biofertilizer (2kg ha-1) & 8.38 \\
\hline $\mathrm{T}_{5}$ & Vermicompost $2.5 \mathrm{t}$ ha- $1+50 \%$ NPK Biofertilizer ( $2 \mathrm{~kg}$ ha- 1$)$ & 7.50 \\
\hline $\mathrm{T}_{6}$ & FYM 10 t ha- $1+75 \%$ NPK + Biofertilizer (2kg ha-1) & 8.75 \\
\hline $\mathrm{T}_{7}$ & Vermicompost $2.5 \mathrm{t}$ ha-1 + Biofertilizer (2 kg ha-1) & 9.14 \\
\hline $\mathrm{T}_{8}$ & FYM 10 t ha-1+Vermicompost 2.5t ha-1 + Biofertilizer (2 kg ha-1) & 9.22 \\
\hline $\mathrm{T}_{9}$ & Vermicompost $2.5 \mathrm{t}$ ha-1 + Biofertilizer (2 kg ha-1) + $75 \% \mathrm{NPK}$ & 7.77 \\
\hline $\mathrm{T}_{10}$ & Vermicompost $2.5 \mathrm{t}$ ha-1+ NPK50\% + Biofertilizer (2 kg ha-1) & 8.34 \\
\hline $\mathrm{T}_{11}$ & FYM $10 \mathrm{t}$ ha-1+Vermicompost 2.5t ha-1 + Biofertilizer (2 kg ha-1) 75\% NPK & 9.46 \\
\hline \multirow[t]{5}{*}{$\mathrm{T}_{12}$} & 100\% NPK (60:80:75 kg ha-1) & 8.45 \\
\hline & F-Test & $\mathrm{S}$ \\
\hline & C.D. at $0.5 \%$ & 0.882 \\
\hline & S.Ed. ( \pm$)$ & 0.427 \\
\hline & $\mathrm{CV}$ & 6.169 \\
\hline
\end{tabular}


Varun Shekhar et al, International Journal of Advances in Agricultural Science \& Technology,

Vol.8 Issue.10, October-2021, pg. 17-28

ISSN: 2348-1358 Impact Factor: 6.057 NAAS Rating: 3.77

5. Economics of different treatments and cost: benefit ratio

\begin{tabular}{|c|c|c|c|c|c|c|c|}
\hline \multicolumn{2}{|r|}{ Treatments } & \multirow{2}{*}{$\begin{array}{c}\text { Yield (q/ha) } \\
120.66 \\
\end{array}$} & \multirow{2}{*}{$\begin{array}{c}\begin{array}{c}\text { Selling } \\
\text { price } \\
(\text { Rs./q) }\end{array} \\
1000 \\
\end{array}$} & \multirow{2}{*}{$\begin{array}{c}\begin{array}{c}\text { Cost of } \\
\text { Cultivation }\end{array} \\
62900 \\
\end{array}$} & \multirow{2}{*}{$\begin{array}{c}\text { Gross } \\
\text { return } \\
120660 \\
\end{array}$} & \multirow{2}{*}{$\begin{array}{c}\text { Net return } \\
57760\end{array}$} & \multirow{2}{*}{$\begin{array}{c}\begin{array}{c}\text { Cost benefit } \\
\text { ratio }\end{array} \\
1.92 \\
\end{array}$} \\
\hline $\mathrm{T}_{0}$ & Control & & & & & & \\
\hline $\mathrm{T}_{1}$ & FYM 20t ha-1 & 292.53 & 1000 & 122900 & 292530 & 169630 & 2.38 \\
\hline $\mathrm{T}_{2}$ & Vermicompost 5 t ha-1 & 325.92 & 1000 & 82900 & 325920 & 243020 & 3.93 \\
\hline $\mathrm{T}_{3}$ & FYM 10 t ha-1 + Vermicompost 2.5 t ha-1 + NPK $25 \%$ & 333.61 & 1000 & 105030 & 333610 & 228580 & 3.18 \\
\hline $\mathrm{T}_{4}$ & FYM 10 t ha-1+50\% NPK+ Biofertilizer (2kg ha-1) & 359.93 & 1000 & 98672.1 & 359930 & 261257.9 & 3.65 \\
\hline $\mathrm{T}_{5}$ & Vermicompost $2.5 \mathrm{t}$ ha-1+50\%NPK Biofertilizer (2 kg ha-1) & 345.41 & 1000 & 78672.1 & 345410 & 266737.9 & 4.39 \\
\hline $\mathrm{T}_{6}$ & FYM 10 t ha-1+ 75\% NPK + Biofertilizer (2kg ha-1) & 337.21 & 1000 & 100811.5 & 337210 & 236398.5 & 3.34 \\
\hline $\mathrm{T}_{7}$ & Vermicompost $2.5 \mathrm{t}$ ha-1 + Biofertilizer (2 kg ha-1) & 375.49 & 1000 & 74400 & 375490 & 301090 & 5.05 \\
\hline $\mathrm{T}_{8}$ & $\begin{array}{l}\text { FYM } 10 \text { t ha-1+Vermicompost } 2.5 \mathrm{t} \text { ha- } 1+\text { Biofertilizer }(2 \mathrm{~kg} \\
\text { ha-1) }\end{array}$ & 420.80 & 1000 & 104400 & 420800 & 316400 & 4.03 \\
\hline $\mathrm{T}_{9}$ & $\begin{array}{l}\text { Vermicompost } 2.5 \text { t ha-1 + Biofertilizer (2 kg ha-1) + } 75 \\
\% \text { NPK }\end{array}$ & 407.20 & 1000 & 80811.5 & 407200 & 326388.5 & 5.04 \\
\hline $\mathrm{T}_{10}$ & Vermicompost 2.5 t ha-1+ NPK50\% + Biofertilizer (2 kg ha-1) & 412.13 & 1000 & 78672.1 & 412130 & 333457.9 & 5.24 \\
\hline $\mathrm{T}_{11}$ & $\begin{array}{l}\text { FYM } 10 \text { t ha-1+Vermicompost } 2.5 \mathrm{t} \text { ha- } 1+\text { Biofertilizer }(2 \mathrm{~kg} \\
\text { ha-1) } 75 \% \text { NPK }\end{array}$ & 445.00 & 1000 & 108672.1 & 445000 & 336327.9 & 4.09 \\
\hline $\mathrm{T}_{12}$ & $100 \%$ NPK (60:80:75 kg ha-1) & 378.05 & 1000 & 71454.3 & 378050 & 306595.7 & 5.29 \\
\hline
\end{tabular}


Varun Shekhar et al, International Journal of Advances in Agricultural Science \& Technology,

Vol.8 Issue.10, October-2021, pg. 17-28

ISSN: $2348-1358$

Impact Factor: 6.057

NAAS Rating: 3.77

\section{CONCLUSION}

The treatment combination $\mathrm{T}_{11}$ [ FYM $10 \mathrm{t}$ ha-1+Vermicompost 2.5t ha-1 + Biofertilizer (2 kg ha-1) 75\% NPK] was appropriate for Carrot in Prayagraj. Conditions for getting maximum growth and yield. Whereas the maximum benefit cost ratio ( $5.24 \& 5.29)$ was found in $\mathrm{T}_{10}$ and $\mathrm{T}_{12}$, but there is need further of investigation to confirm the result at various locations.

\section{REFERENCES}

[1]. Handelman. G.J. 2001. The evolving role of carotenoids in human biochemistry. Nutrition, 17:818-822.

[2]. Kochhar, S. L (2011). Economic Botany in the Tropics. Fourth Edition, pp245-246. Macmillan.

[3]. Mehwish Kiran, Muhammad Saleem Jilani, Kashif Waseem and Sarfaraz Khan Marwat (2016). Response of Carrot (Daucus carota L.) Growth and Yields to Organic Manure and Inorganic Fertilizers . American-Eurasian J. Agric. \& Environ. Sci., 16 (6): 1211-1218

[4]. (Tiwari et al., 2012). Integrated nutrient management on growth, yield and quality of carrot. Karnataka J. Agric. Sci. 23 (3): $542-543$

[5]. Singh D.P., Kumar Sanjay, Maji Sutanu and Pandey Vijay Kumar (2017). Studies on integrated nutrient management on growth, yield and quality of carrot (Daucus carota L.). International Journal of Agriculture Sciences, 8:(51), pp.-2187-2188 